\title{
Vitamin D deficiency in an older, northern population - the perfect storm on the horizon?
}

\author{
Helena Scully $^{1}$, Eamon Laird ${ }^{2}$, Martin Healy ${ }^{3}$, Kevin McCarroll ${ }^{1}$ and J. Bernard Walsh ${ }^{1}$ \\ ${ }^{1}$ Mercer's Institute for Research on Ageing, St. James's Hospital, Dublin 8, Ireland, \\ ${ }^{2}$ School of Medicine, Trinity College Dublin, Dublin 2, Ireland and \\ ${ }^{3}$ Department of Biochemistry, St James's Hospital, Dublin 8, Ireland
}

\section{Abstract}

Introduction: Vitamin D deficiency (VDD) is detrimental to bone health, playing an intrinsic role in osteoporosis and rickets. Recently it has been linked to morbidities including inflammation, cardiovascular disease and cognition. The majority ( $90 \%)$ of vita$\min \mathrm{D}$ is obtained by the action of UVB light on the skin, this is reduced in northern latitudes $\left(>42^{\circ} \mathrm{N}\right)$, by SPF, darker skin tone, and ageing. Bioavailability is affected by internal factors including obesity and malabsorption. Many developed populations are becoming older and more overweight. It is essential to ascertain the extent of VDD to predict further trends. The aim of this study is to investigate vitamin D status in a population of GP requested samples within the St James Hospital (SJH) catchment area.

Materials \& Methods: The SJH catchment area sits at northerly latitude $\left(53^{\circ} \mathrm{N}\right)$ and includes rural and urban environments (Dublin City, Dublin County and County Kildare) of various socioeconomic groups. An estimated $60 \%$ of the population are overweight/ obese, with $20 \%$ aged 65 and over. A data-set of total $25(\mathrm{OH}) \mathrm{D}$ concentrations (measured by LC-MS/MS) was created from the SJH laboratory information system from GPs requests between the years 2014-2016. Results were tabulated according to geometric mean values for vitamin $D$ in each postal district with percentage of samples deficient $(<30 \mathrm{nmol} / \mathrm{L})$, insufficient $(30-50 \mathrm{nmol} / \mathrm{L})$, and sufficient $(>50 \mathrm{nmol} / \mathrm{L})$. This data was further stratified by age $(18-50,>50$ years) and socioeconomic status and analysed by ANOVA.

Results: A total of 15,483 GP samples were received for vitamin D requests in the time period studied. Preliminary results indicate VDD in $15.2 \%$ of the population, with $22.4 \%$ insufficient. The lowest socioeconomic areas (Dublin 8 and Lucan postal district) were the most consistently deficient $(23.5 \%$ and $20.4 \%$, respectively). The geometric mean $25(\mathrm{OH}) \mathrm{D}$ concentration in the total population was $56.2 \mathrm{nmol} / \mathrm{L}$ (SD 31.5), with those age 18-50 years more lively to be deficient than those $>50$ years $(\mathrm{P}<0.0001)$.

Discussion: This study indicates that VDD remains prevalent across age and location groups at a northern location. Current trends in developed populations, such as the obesity epidemic and ageing populations, may increase rates of deficiency and burden of diseases. With the extent of vitamin D deficiency becoming better understood, its contributing factors require greater evaluation to understand the potential consequences in the population. As such, further analysis and investigations are planned to explore factors contributing to VDD in this cohort.

\section{Conflict of Interest}

HS is a Research Fellow funded by Glanbia PLC. Glanbia had no role in study design, data collection and analysis, decision to publish, or preparation of the manuscript. 